\title{
EL ENTRE-LUGAR COMO UN PENSAMIENTO DEL RIESGO

\author{
Entrevista a Silviano Santiago
}

Alejandro Fielbaum

Universidad Adolfo Ibáñez

Rebeca Errázuriz Cruz

Universidad de Chile / Universidad de Talca

La obra de Silviano Santiago (1936) constituye una de las propuestas más novedosas de la crítica brasileña de las últimas décadas. Con una amplia obra que ha transitado diversos géneros como la poesía, la novela y el ensayo, el autor de "El entre-lugar del discurso latinoamericano" "ha creado un pensamiento que busca romper con la dependencia de las metrópolis sin por ello caer en un nacionalismo o latinoamericanismo a ultranza. La originalidad de sus reflexiones consiste justamente en la búsqueda constante por abrir la posibilidad de pensar la cultura brasileña, latinoamericana o las culturas marginales desde perspectivas que rompen con lugares tradicionales y concepciones ya establecidas, para iluminar aspectos invisibilizados que permiten desmontar las nociones usuales de identidad. Algunas de sus obras más destacadas son los libros de ensayos Uma literatura nos trópicos. Ensaios sobre dependência cultural (1978), Vale quanto pesa. Ensaios sobre questões político-culturais (1982), Nas malhas da letra (1989), O Cosmopolitismo do Pobre. Crítica Literária e Crítica Cultural (2004) y As Raízes e o Labirinto da América Latina (2006) ${ }^{2}$; junto con las novelas Em liberdade (1981), Stella Manhattan (1985) y

1 Traducido al español por Mary Luz Estupiñán y Raúl Rodríguez, quienes editaron una antología de los principales ensayos de Silviano Santiago. Cf. Silviano Santiago, Una literatura en los trópicos. Ensayos de Silviano Santiago. Concepción: Editorial Escaparate, 2012.

Existe una traducción al español realizada por Mónica González García: Cf. Silviano Santiago, Las raices y el laberinto de América Latina. Buenos Aires: Editorial Corregidor, 2013. 
Viagem ao México (1993); entre otras. Recientemente, Silviano Santiago ganó el Premio Iberoamericano de Letras José Donoso 2014.

La siguiente entrevista tuvo lugar en el contexto del coloquio internacional Entrelugar y Traducción, organizado en conjunto por el Departamento de Filosofía de la Universidad Metropolitana de Ciencias de la Educación, el Doctorado en Estética y Teoría del Arte de la Universidad de Chile y el Magíster en Literatura Chilena y Latinoamericana de la Universidad de Santiago, a principios de agosto del 2013. En esa ocasión conversamos con el autor acerca de ciertas ideas que cruzan su obra, como el entre-lugar y el cosmopolitismo, en relación con el campo cultural brasileño, la teoría postcolonial y el panorama actual de la producción del saber.

RE-AF: Tanto en su obra critica como en su obra literaria podemos observar ciertas líneas mayores que permiten detectar un esfuerzo y una necesidad de pensar desde lo que usted llama el "entre-lugar". Asimismo, el afán por ocupar el lugar de otro, por hablar desde o a partir de la voz de otro, como sucede en sus obras Em Liberdade (1981) y Viagem ao México (1995), es un afán que reconoce el lugar de la escritura como una doble prisión, tal como usted lo menciona en "O ficcionista como tradutor";; pero en el reconocimiento de esa prisión transformada en lugar de creación y de escritura se opera una transgresión, como bien señala usted en "Eça autor de Madame Bovary". Esta transgresión es mencionada por usted en su ensayo sobre el entre-lugar como un acto de habla o de escritura que es siempre un hablar contra, un escribir contra. Mi pregunta es cuál es el lugar que usted le asigna a la transgresión como práctica creadora en la literatura latinoamericana y en la crítica, y cómo dar lugar a esa transgresión al interior de un medio universitario crecientemente especializado, que parece haber renunciado a esa posibilidad para plegarse a la retórica de la producción de conocimiento "académico".

SS: Se trata de una pregunta complicada, por lo que prefiero responder por partes. En primer lugar, tengo que decir que el entre-lugar debe ser comprendido, inicialmente, en la perspectiva de lo que nosotros llamamos estudios postcoloniales. La conferencia donde se desarrolla esa idea por primera vez fue escrita para hablar, por un lado, sobre la condición de literaturas que fueron escritas en naciones colonizadas por Europa -en el caso latinoamericano, por España y Portugal-y, por otro lado, para indagar y develar en qué sentido esta producción colonial podría tener significado para nosotros en la actualidad. En otras palabras, no se trata de leer esta producción ni en el lugar europeo ni en el lugar nacional, sino en el entre-lugar. Ese fue el sentido básico de mi idea en aquella época: que el entre-lugar posibilitaría un juego de significaciones que no están limitadas por lo nacional, lo que sería algo peligroso ya que puede conducir a un nacionalismo estrecho y autosuficiente, que da la espalda a lo extranjero; ni tampoco por el lugar de

3 Texto leído durante el coloquio internacional Entrelugar y traducción, traducido y publicado en la Revista Escrituras americanas no. 2, 2013. 11-16. 
lo europeo, ya que estaríamos tan solo insistiendo en la fuerza de la fuente y en la fuerza de la influencia, vale decir: en el silencio de los colonizados. En otras palabras, esa producción sería ibérica pues estaría imitando los modelos europeos.

Entonces, lo entre surge como una postura que llamaría alternativa, ya que los estudios literarios estaban alineados con un lado o con otro. Pero surge también, más que como alternativa, como una práctica de lectura para repensar la literatura comparada. Me parece que ese es el segundo paso, repensar qué es y qué ha sido esa disciplina, ciertamente europea, ciertamente eurocéntrica, que llamamos literatura comparada. Esto nos lleva a reconsiderar las producciones posteriores a la independencia (en el caso de Brasil, después de 1822) junto con las producciones propiamente coloniales (antes de 1822). Dentro de esa producción post independencia, puede darse entonces una nueva lectura y el ejemplo que di para no caer en un nacionalismo estrecho no fue uno brasileño sino portugués, el de Eça de Queirós. La mirada del entre-lugar permite leer el siglo XIX y permite también una relectura del modernismo brasileño 4 que surge después de 1922 y que, no por casualidad, se sitúa en el contexto del centenario de la independencia brasileña. Y allí, en la lectura del modernismo brasileño, obviamente ya se percibe una aproximación más nítida de la idea de entre-lugar con el ideario vanguardista europeo propiamente tal.

En segundo lugar está lo relativo a la creación literaria. No me gusta mucho asociarla, pero sí aproximarla, al ensayismo. Hay una diferencia entre ese ensayismo, que es nítidamente postcolonial, y mi creación literaria, en la que existe un deseo mayor de diálogo con el modernismo brasileño de los años 1920, que ya no está tan marcada por el postcolonialismo y sí por el postmodernismo. El modernismo brasileño presentaba para mí, como joven escritor, la posibilidad de una tradición compatible con mis deseos y ansiedades de escritor en los años 1970. Eran autores que yo admiraba. Retomando lo escrito por Roland Barthes en $S / Z^{5}$, la producción modernista en Brasil me generaba un deseo de reescribir lo ya escrito.

En ese sentido, si quizás el gesto dominante del pensamiento postcolonial del entrelugar es escribir contra, el de la creación literaria no es ese, sino el de escribir con afecto. Pero no para imitar. Yo no quería imitar a Graciliano Ramos, por lo que debía entonces buscar una brecha, un lugar entre. Si hubiera querido imitarlo hubiese escrito un ensayo sobre Graciliano Ramos, no una creación novelística, y esa creación debía ser pensada y escrita desde ese lugar entre, justamente situado después del pasado reciente y terrible de la represión, que incluyó torturas, interrogatorios, persecuciones, la misma prisión o el exilio. Se trata de un momento histórico en que comienzan las ideas de apertura ideológica

$4 \quad$ En la literatura brasileña, el modernismo, que se inicia con la Semana de Arte Moderno de São Paulo en 1922, equivale a los movimientos de vanguardia hispanoamericanos. Es importante no confundirlo con el modernismo hispanoamericano.

"Pourquoi le scriptible est-il notre valeur? Parce que l'enjeu du travail littéraire (de la littérature comme travail), c'est de faire du lecteur, non plus un consommateur, mais un producteur de texte". En Barthes, Roland, S/Z. Essai sur Sarrasine d'Honoré de Balzac. Paris: Éditions du Seuil, 1970, p. 10. 
en Brasil, entonces, de lo que se trata es de retirar del discurso político la cuestión de la prisión para enfocarse en la de la libertad, en qué hacer cuando se está en libertad. Se trataba de trabajar eso de forma aproximada, pero diferente. La elección de un autor de la década del treinta no fue casual. No quise escoger un caso de 1964. Escogí un caso de represión de esos años treinta y no de los sesenta o setenta, porque tuve el interés de que la novela tuviese un valor simbólico o alegórico, por así decirlo, que pudiera mostrar que en Brasil es natural -con todas las comillas del caso- la represión a los intelectuales que no son conservadores. Entonces, de forma indirecta, simbólica o alegórica, según se lea la novela, estaba hablando de los años sesenta y setenta. Por otro lado, en la segunda parte de la novela, me intereso por un episodio histórico semejante, ocurrido durante el periodo colonial en Minas Gerais. Hablo del acontecimiento que fue la inconfidencia mineira ${ }^{6}$ y del "suicidio" de Cláudio Manoel da Costa, y los comparo, en la novela, a lo sucedido con el periodista Vladimir Herzog, "suicidado" por la represión en 1975. Mi interés en la novela Em liberdade era dramatizar un panel amplio del papel del intelectual brasileño en la política y no concentrarme simplemente en los acontecimientos ocurridos durante los años 1960/1970. Para eso fue necesario construir un lugar entre al interior de los discursos autobiográficos de los jóvenes revolucionarios de los años 1960, ya que estos discursos, como por ejemplo O qué é isso companheiro? (1979), de Fernando Gabeira, pueden tratar muy bien los acontecimientos de los años que relatan, pero no poseen esa amplitud que la novela sí puede tener. Digo todo esto para remarcar que el entre posee una polisemia que me gustaría que se preservara, sin un sentido estrecho que limitase el juego, entendido en términos derridianos; y que debe ser circunscrito con cierto cuidado para que no se vuelva algo caricaturesco que me transforme en algo así como el pensador del entre.

¿Y cómo considera ese sentido transgresor, dentro de la crítica? ¿Puede tener lugar hoy dentro de la academia y sus criterios -producidos muchas veces desde las metrópolis-para distinguir y jerarquizar tipos de conocimientos, los que parecen ir en desmedro de un género como el ensayo?

Creo que para eso tendríamos que remitirnos, en la reciente respuesta, al primer sentido de lo entre, pensando cómo se podría plantear hoy en la discusión postcolonial. Sería difícil para mí hacer un panorama completo del campo latinoamericano, pero en el espacio brasilero puedo ser más específico. En mi caso particular, estoy trabajando la idea de la transgresión desde una relectura de la antropofagia ${ }^{7}$, que ciertamente debe ser diferente

$6 \quad$ La Conspiración Minera fue un movimiento de revuelta contra el dominio portugués en la región aurífera de la entonces provincia de Minas Gerais. La revuelta fue abortada por la corona portuguesa en 1789. El poeta Cláudio Manoel da Costa, uno de los principales rebeldes, fue dado como suicida por la historia, pero en realidad debe haber sido "suicidado" en secreto, antes del inicio de la investigación policial.

Teoría desarrollada por Oswald de Andrade, en particular en sus manifiestos literarios Manifesto Poesia Pau Brasil (1924) y Manifesto Antropófago (1928). 
de la concepción original de la antropofagia que está en Oswald de Andrade, pues si no no haría más que monumentalizar, de forma imitativa, su obra. Por el contrario, intento llevar la antropofagia al siglo XXI, tratando cuestiones del pasado pero ya no en el contexto del pasado, sino del presente. En mi caso, intento retomar una ligera nota cosmopolita que existía ya en mis estudios de los años setenta y ochenta, en el contexto del nuevo milenio y ante la globalización. Ahí, intento abrir una brecha desde el cosmopolitismo de la élite para oponerlo al cosmopolitismo del pobre, que me permite aproximar la teoría postcolonial de los años setenta y ochenta con los movimientos diaspóricos del nuevo siglo. En ese sentido, por ejemplo, trabajo con las ideas de Stuart Hall, quien no estaba en mi bibliografía de las décadas previas.

En la actual situación, son los antiguos países colonizadores los que, por así decirlo, están siendo colonizados por los excolonos. No tanto desde América Latina porque ya tenemos una situación económica y social que, sin ser la ideal, ha mejorado; pero desde los países africanos y asiáticos donde la migración en dirección a los países colonizadores ha aumentado. Entonces, desde ahí, me parece que nosotros podemos lanzar otras redes para la disciplina de la literatura comparada, ya que poco podríamos comprender la nueva producción proveniente de los países africanos, árabes o asiáticos desde una visión que sea a priori exclusivamente latinoamericana o exclusivamente circunscrita a Europa. Esa sería una forma de verlo, podemos continuar trabajando pero con el coraje de ensanchar los corpus del trabajo.

En el caso latinoamericano en particular, quizás no se trata solo de analizar los movimientos diaspóricos sino también la inserción de la cultura indígena en la cultura nacional canónica y, especialmente en el caso de Brasil, de la cultura africana. Esta atención es necesaria tanto en sus formas, digamos, más "puras", ligadas a las contribuciones de grupos indígenas o africanos, como en manifestaciones como una literatura afrobrasileña actual, o una literatura indígena actual. Ahí, quizás, Oswald de Andrade -en la antropofagiasería menos importante que Mário de Andrade y su novela Macunaíma (1928), a quien se puede entonces recuperar estratégicamente y continuaríamos de cierta manera hablando de antropofagia.

También surgen en el espacio latinoamericano otras cuestiones que hoy pueden ser discutidas. Pienso, al menos, en dos: la ciudadanía y la democracia, temas que posibilitan también una revisión de la teoría antropofágica, no solo la de los años veinte y treinta, sino también la forma cómo retorna en los años setenta, ochenta y noventa, en particular a partir del Tropicalismo y del Teatro Oficina, liderado por José Celso Martinez. En esa discusión existe un elemento que aún no he aprehendido muy bien y que simplemente lanzo en este momento, que es la posibilidad de trabajar desde los afectos. Ya no hay necesidad de hostilizar a Europa, podemos establecer un diálogo no desde presupuestos coloniales violentos -y es lo que hago en mi trabajo sobre Adriana Varejão-, sino a partir de una política de la preferencia por el afecto, sin hostilidad con Europa. Yo preferiría, como Bartleby, que nosotros no fuéramos eurocéntricos, sería mucho más interesante para nosotros y para los europeos. Nuestro diálogo sería más fraterno, más afectuoso. Entonces, existe algo que yo no sé muy bien aún cómo tratar, pero diría con toda sinceridad que eso es un elemento nuevo. 
Me parece, por otro lado, que no hay necesidad de quedarse en los modelos pasados, por ejemplo, en el modelo norteamericano de investigación sobre la cuestión homosexual y de la producción universitaria correspondiente. Me parece que es cuestión de ir haciendo dislocamientos y no repeticiones, de encontrar brechas significativas y trabajarlas de manera creativa, inteligente.

Retomando esta idea de los diálogos a partir de los afectos en contraposición al "hablar contra", en el campo intelectual brasileño su figura como crítico ha sido contrapuesta a la de la llamada escuela "uspiana", principalmente en contraste con la figura de Roberto Schwarz. Sin embargo, es posible leer vasos comunicantes entre su trabajo y la obra de Antonio Candido, como lo hace Célia Pedrosa. Me parece que Flora Süssekind hace otro tanto en su lectura de la figura de Antonio Candido. Desde su posición ¿cree usted que esta llamada crítica uspiana ha despojado a la obra de Candido de un posible carácter transgresor?

De partida, creo que no hay antagonismo, en el sentido guerrero, sino más bien una especie de complementariedad, entre mi trabajo y el de Candido. Y considero algo exagerado ese diagnóstico sobre la Universidad de São Paulo, ya que hay trabajos extraordinarios en la escuela uspiana.

Lo que diría es que tendríamos que analizar con mucho cuidado -y enfatizo ahí los estudios sobre cosmopolitismo que estoy realizando actualmente-, la formación de la USP, que es algo sobre lo que nadie habla ${ }^{8}$. Entonces, antes de hablar de Candido o de una escuela literaria uspiana, habría que hablar de cómo la USP fue formada. Esta universidad fue formada por jóvenes profesores franceses, quienes eran eurocéntricos en su enseñanza, pero no así en su investigación sobre Brasil. Creo que lo que a la USP le pesó y le sigue pesando fue su apego a la enseñanza de esos profesores franceses, sin notar que Claude Lévi-Strauss pasó todo su tiempo en Brasil estudiando a los indígenas, y Roger Bastide, a los afrobrasileños. Daban clases desde los presupuestos europeos de las disciplinas, pero hacían un trabajo de campo no eurocéntrico. Creo que fue muy difícil para la USP aceptar esta paradoja, ya que lo más importante era la formación de los estudiantes brasileños, realizada de manera muy disciplinar por intelectuales europeos.

Por ello la crítica que yo haría es que ese carácter disciplinario del trabajo de los críticos literarios formados por USP - que debe ser aplaudido y que ciertamente ha sido un aporte extraordinario porque en Brasil antes de ellos no había crítica literaria- resulta un tanto intolerante con otras disciplinas, salvo con la sociología. Con la antropología, por ejemplo, no hubo diálogo. Entonces, dentro de esa perspectiva, la de la USP es todavía una crítica eurocéntrica. Creo que ahí hay también un problema-que no debe trabajarse con insolencia sino con mucha delicadeza- y es que Roberto Schwarz es un hombre europeo, no brasileño, y que resulta muy difícil entonces que no haya tenido

8 Recientemente, en el mes de octubre de 2014, nuestro entrevistado publicó el ensayo "La literatura brasileña desde una perspectiva poscolonial - un relato", presentado en un simposio en la Universidad Nacional de Tres de Febrero (Buenos Aires). 
esa marca europea, tal como yo no dejé de trabajar a partir de mi propia experiencia brasileña en Europa o Estados Unidos. El sentido disciplinar de la crítica literaria que surge en la USP posee una nítida marca eurocéntrica, que tuvo la gran cualidad de dar un estatuto de mucha seguridad a la disciplina de la teoría literaria y a la de la historia de la literatura en Brasil, que tiene como mayor ejemplo la Formação da literatura brasilera de Antonio Candido.

Hay que sumar, además, otra manifestación eurocéntrica, y es que ellos tienen un cierto recelo ante la vanguardia y a trabajar con lo contemporáneo, lo que obviamente no tiene que ver con una cuestión de inteligencia, sino con que no tienen el instrumental riguroso para analizar las producciones literarias nuevas y poco convencionales, ante las cuales se sienten, creo yo, algo intimidados. Creo que esa timidez teórica y disciplinar lleva a la crítica de la USP a preocuparse más por el pasado. Por ejemplo, los estudios más importantes de Schwarz, que ciertamente son una excelente contribución, son sobre autores del siglo XIX. Y de este modo pueden trabajar ese corpus con mucha seguridad. Si tomamos "Las ideas fuera de lugar", de Roberto Schwarz, vemos que es sin duda una gran contribución, pero es también dependiente de una lectura marxista del siglo XIX.

Pero, volviendo a Antonio Candido, tengo la impresión de que la de él es una obra mucho más flexible, mucho más transgresora, en términos disciplinares, que aquello en lo cual se transformó la crítica uspiana. Me llama la atención ese contraste, que la crítica uspiana no ve ese carácter transgresor y, en lugar de seguir ese ejemplo, quedó presa de la teoría, presa de la disciplina.

Ah, claro, sí, en ese sentido, así es.

Quizás esa tradición transgresora la retoma mucho mejor usted que sus discípulos directos.

No sé si yo, necesariamente, pero muchos de mis discípulos sí, como, por ejemplo, Flora Süssekind, particularmente en algunos de sus trabajos aún no publicados y que me parece que serán definitivos. Uno versa sobre el poema épico romántico brasilero y sus relaciones con la pintura, y otro sobre un contrapunto entre Sousândrade y José Martí en Nueva York, los que muestran otro siglo XIX, yendo más allá de lo brasileño en sentido estrecho: por un lado, al plantear la relación de las letras con las artes; por otro, al investigar la relación entre dos grandes intelectuales latinoamericanos en Manhattan. Ella es una autora que en algún punto sigue su senda sin perjuicio de que yo, entre muchos otros, fui uno de sus profesores, insistiendo siempre en que ella, al igual que el resto de mis estudiantes, debía tener una mirada propia.

A propósito de su debate con Roberto Schwarz, pensando en que hoy, en el contexto de la globalización, la crítica latinoamericana pareciera a veces estar entrampada entre una posición identitaria que es más o menos esencialista o bien una posición de apertura a la globalización que muchas veces termina siendo cómplice del neoliberalismo ¿desde dónde cree usted que se podría pensar políticamente el lugar del entre-lugar en un mundo en el cual, desde una lectura 
apresurada e ingenua, se podría decir que todo ha devenido entre-lugar, por las relaciones de migraciones y el progresivo desdibujamiento de las fronteras nacionales duras versus la posición de la identidad que termina siendo a veces excesivamente nacionalista?

Me parece que tendría que retomar mi primera respuesta: el entre-lugar debe ser leído desde las teorías postcoloniales y tomando el debido cuidado para que éste no se confunda con las formas identitarias del nacionalismo. El entre está allí justamente como el mejor antídoto ante ese nacionalismo estrecho, que da la espalda a lo extranjero, sobre todo en cultura, en arte, en educación o en la formación de los jóvenes; y que obviamente tiene la actitud de un contra que no desconoce el hecho de que todos estamos, en primer lugar, en un continente y en un mundo. Esa sería una primera actitud. La otra actitud, de la que ya hablé y que es un poco caricaturesca, es aquella que asimila el entre lugar a todo y a cualquier cosa. Es una lectura equivocada.

Esas distinciones son hechas de manera muy nítida en la misma teorización del cosmopolitismo, no a partir de un cosmopolitismo de cuño romano, sino de un cosmopolitismo que es pensado a partir de los movimientos diaspóricos. Y allí tenemos varios pensadores de formación marxista, pero no marxista en un sentido eurocéntrico, cuyo mejor ejemplo es el caso del jamaicano Stuart Hall. Si existe un pensamiento identitario desde el entre-lugar, éste sería muy cercano a lo que Stuart Hall dice acerca la cuestión del viaje, que ya existe en Roger Bastide cuando analiza a los afrobrasileños. Bastide dice que el blanco no consigue entender que el negro pueda viajar del candomblé a la fábrica y ser obrero. Es un viaje que el jamaicano también realiza cuando sale del Caribe y va a vivir en Londres. El viaje perpetuo es la posibilidad de que dentro de un mundo masivamente blanco y elitizado, como el europeo, se pueda crear un lugar allí adentro donde el jamaicano tiene sus referencias africanas muy presentes.

Entonces, el entre-lugar, independiente de que pueda ser mal entendido o sobre simplificado, cuestión que ya no depende de mí, y más allá de que no sea perfecto, es un pensamiento que se sostiene como una teoría no conservadora, no liberal ni neoliberal, sino que intenta comprender una cosa que es muy difícil de comprender que es lo contemporáneo. Para comprender lo contemporáneo no basta un pensamiento enciclopédico, que no avanza, que no es de vanguardia; que da cuenta de lo que está ocurriendo hoy en día pero no lanza al lector hacia nuevas cuestiones, hacia nuevos puntos críticos. Lo que es muy importante en el entre-lugar es que es una teoría del riesgo, del peligro, no es una teoría de la tranquilidad y eso es lo que me parece más importante, es una teoría donde estamos obligados a arriesgarnos, a construir un camino propio y abrir espacios de discusión, que a veces pueden ser muy fructíferos y a veces no, porque no hay garantías.

Y eso está relacionado con lo que hablábamos hace un momento sobre el carácter transgresor, con la necesidad de romper ciertos lugares de discusión, ciertas teorías o pensamientos que se transforman en prisiones.

Es eso mismo. Hace un momento conversaba con uno de los participantes de mi mesa, Andrés Claro, acerca de que hoy en día no existe un buen discípulo de Jacques Derrida, 
porque todos están hipnotizados por el pensamiento del propio Derrida. A diferencia de Derrida, que hizo relecturas del pensamiento de Lacan, de Lévi-Strauss, de Michel Foucault, en las cuales siempre existía una expansión de las fronteras, de las ideas. Y la necesidad actual es justamente esa, la de crear una teoría como riesgo.

\section{En ese sentido, ¿cómo evaluaría la relación que ha habido entre la deconstrucción} de Derrida y los estudios latinoamericanos?

Me parece que lo más importante en Derrida es la cuestión de la lectura. Habría que pasar mucho tiempo discutiendo en qué se transformó la lectura después de Derrida y después del postestructuralismo. Y esto también es importante al interior del pensamiento latinoamericano, pues nos impulsa a leer de manera diferente. Y me parece que esa es otra de las cuestiones que se pueden examinar en la crítica uspiana en contraste con Antonio Candido, y es que ellos no intentaron leer de manera diferente. Nuevamente es una teoría muy disciplinar, que no intenta relacionarse con cuestiones como las artes o el cine, que no abre los horizontes hacia nuevas posibilidades de lecturas, sino que tiene miras muy estrechas. Yo, por ejemplo, trabajé como crítico de cine durante muchos años y aprendí mucho sobre cine y sobre teoría cinematográfica. Eso me permitió diferenciar entre tipos de montajes como el de André Bazin, que privilegia el plano-secuencia, versus el de Eisenstein, que trabaja con un montaje de choque. Por eso no se puede hablar de montaje sin definirlo a priori. Adriana Varejão, por ejemplo, trabaja en sus obras de arte con un tipo de montaje más cercano al de Eisenstein, lo que a su vez es una contribución hecha en Brasil por la poesía concreta, que a su vez toma la idea de Ezra Pound del ideograma. Es eso lo que me parece importante hacer, posibilitar nuevas lecturas, en el caso de la poesía concreta, más allá de su lugar en la tradición literaria brasileña, lo importante es mostrar que era en su proyecto una poesía multi-artística, donde había una propuesta que no era literaria en el sentido estrecho de la palabra.

Esa me parece que es la gran contribución de Derrida, la de abrir la posibilidad de nuevas lecturas. Derrida tenía la ventaja de ser un lector extraordinario, no conozco mejor lector que Derrida. Para dar un ejemplo, tenemos su lectura de los diálogos de Platón , que ya habían sido ampliamente leídos e interpretados y, sin embargo, él fue capaz de leer allí cuestiones de una novedad absoluta.

En segundo lugar, otra cosa que me parece importante es que las lecturas de Derrida -y esto es algo que le ha causado problemas hasta el día de hoy- eran siempre lecturas que buscaban desentrañar los espacios en blanco de las teorías ajenas, lo que permite no monumentalizar las obras leídas y admiradas. Por ejemplo, en el caso de Machado de Assis, se vuelve un autor monumentalizado si leemos toda su obra desde Dom Casmurro, y con ello Machado de Assis pierde su riqueza, gana forma, pero pierde fuerza, esa noción de fuerza nítidamente nietzscheana. Eso es admirable, con Derrida se aprende a leer sin hacer una mera repetición ni hacer simplificaciones muy grandes.

9 Véase "La Pharmacie de Platon". En Jacques Derrida La dissémination. Paris: Seuil, 1972. 
Como última pregunta, sus trabajos más recientes abordan la temática cosmopolita desde una perspectiva literaria, recuperando el antiguo vínculo entre cosmopolitismo y literatura que hoy no parece central en el debate europeo que puede leerse en Ulrich Beck, Jürgen Habermas, Thomas Pogge, etc. ¿Por qué cree que en el pensamiento europeo, después de Derrida, ese vínculo parece haberse abandonado? ¿Qué relación cree que existe, por el contrario, entre el pensamiento latinoamericano y el cosmopolitismo y qué papel juega alli la escritura literaria?

Bueno, responder algo así da para un libro, pero puedo al menos mencionar algunos puntos que me parecen importantes. En primer lugar, tenemos el surgimiento de la literatura mundial, habría que pensar por qué y cómo surge, justamente en el momento en que los nacionalismos europeos estaban a flor de piel. Por otro lado, habría que estudiar la historia de la literatura comparada, pues la literatura comparada, a priori, ya es cosmopolita. Esa idea, la del cosmopolitismo, para ser sincero, surgió de Antonio Candido, surgió del prefacio de la Formação da literatura brasileira, donde dice que quien quiera conocer bien la literatura brasileña, no basta con que conozca la literatura en lengua portuguesa, porque la literatura portuguesa misma es una rama pobre en el árbol de la literatura. Cuando Candido ganó el Premio Camões yo estaba en Portugal y el titular del diario era el siguiente "Crítico brasileño desconocido gana el premio Camões". Esa es mi gran deuda con Candido, esa es la deuda del entre-lugar con Candido. Me parece que Formação da literatura brasileira es un libro de una importancia extraordinaria, es un libro de 1959 y podemos ver su diferencia con A literatura no Brasil, de Afrânio Coutinho, publicado en la misma época y que está escrito a partir de una división por estilos de épocas, como el realismo, el naturalismo, el parnasianismo, etc., que es una estructura histórica/crítica pobre. Lo que sería interesante discutir es el concepto de formación en Candido, en particular a partir de la revolución de internet, pero eso es algo que no se hace. Y allí un libro que sería indispensable para discutir la cuestión de la formación es el de Lyotard, La condición postmoderna, que tiene dos capítulos sobre la formación a partir de las nuevas formas de producción del saber en las sociedades informatizadas. No vamos a discutir sobre el secuestro del barroco ${ }^{10}$, que dentro de esa propuesta no tendría sentido, en cambio sí reconozco el mérito de Roberto Schwarz por su trabajo sobre Machado de Assis, que es el gran ausente en Formação da literatura brasileira. El problema es que estos estudios se han quedado un poco en el pasado, lo que en el caso de Candido se comprende, pues debe tener a estas alturas unos 95 años. Roberto y yo tenemos 76, hay entonces una diferencia de veinte años que debería ser significativa. 